\title{
Flare occurrence and the spatial distribution of the magnetic helicity flux
}

\author{
P. Romano ${ }^{1}$ and F. Zuccarello ${ }^{2}$ \\ ${ }^{1}$ INAF - Osservatorio Astrofisico di Catania, via S. Sofia 78, 95123 Catania, Italy \\ e-mail: prom@oact.inaf.it \\ 2 Dipartimento di Fisica e Astronomia - Sezione Astrofisica, Universitá di Catania, via S. Sofia 78, 95123 Catania, Italy
}

Received 29 June 2011 / Accepted 13 September 2011

\begin{abstract}
Context. The accumulation of magnetic helicity via emergence of new magnetic flux and/or shearing photospheric motions is considered an important tool for understanding the processes that lead to eruptive phenomena.

Aims. We highlight a specific aspect of the magnetic helicity accumulation, providing new observational evidence of the role played by the interaction of magnetic field systems that are characterized by opposite signs of the magnetic helicity flux in triggering solar eruptions.

Methods. The amount of magnetic helicity injected into the corona through the photosphere in a sample of active regions (ARs) during their passage across the solar disk was measured by inferring the apparent motion of photospheric footpoints of magnetic field lines from a time series of MDI full-disk line-of-sight magnetograms. The temporal variation of the maps of magnetic helicity flux was analysed by measuring the fragmentation of the patches that are characterized by the flux of magnetic helicity. The temporal correlation between the number of these patches and the flare and coronal mass ejection (CME) occurrence has also been studied. Results. The fragmentation of the patches singled out in the maps of the magnetic helicity flux provides a useful indication of the evolution of the AR complexity. The more fragmented the maps of the magnetic helicity flux are, the higher is the flare and CME frequency. Moreover, most of the events occur for low values $(\sim 3 \div 17)$ of the difference of the number of patches with opposite signs of magnetic helicity flux.

Conclusions. These results indicate that not only the accumulation of magnetic helicity in the corona, but also its positive and negative fragmentation and distribution should be taken into account to provide a more confident indication of AR complexity and flare/CME productivity. In particular, the interaction of magnetic systems characterized by opposite sign of magnetic helicity flux may be responsible for many observed eruptions.
\end{abstract}

Key words. Sun: activity - Sun: flares - Sun: magnetic topology

\section{Introduction}

Many statistical studies are aimed at investigating the relationship between the transport rate of magnetic helicity in the solar atmpsphere and flares or coronal mass ejection (CME) occurrence. Nindos et al. (2003) computed the magnetic helicity injected into the corona by the photospheric horizontal motions in six solar active regions (ARs) associated with halo CMEs by using the method described by Chae et al. (2001). Nindos et al. (2010) computed the velocities using the local correlation tracking (LCT) method (November \& Simon 1988) and found a broad consistency between the helicity injected into the corona and the helicity carried away by the CMEs with the magnetic cloud (MC) helicity computations as proxies to the CME helicity. The helicity flux density maps resulting from Chae's method (the so called $G_{A}$ maps) are contaminated by spurious bipolar signals that may mask the real spatial distribution of helicity injection. Pariat et al. (2006) applied a better proxy to the helicity flux density calculation (the so-called $G_{\theta}$, proposed by Pariat et al. 2005) to the same set of data studied by Nindos et al. (2003). Although they still used the LCT method, they obtained a much more homogeneous pattern for all ARs in the maps of helicity injection because they were able to partially remove the fake polarities.
We note that ideally one obtains the same helicity injection rate, no matter what helicity flux density proxy one uses (see Pariat et al. 2006).

Another important contribution in understanding the relationship between the magnetic helicity and the eruptions occurring in the solar atmosphere was provided by some case studies. In particular, the deeper investigation of the helicity budget and the evolution of a single event as well as the knowledge of the topology of the AR where the event occurred allows one to better understand the particular conditions that bring magnetic field systems to interact and to release energy during eruptive events. Recently, several studies have shown that some eruptions occurred in regions that are characterized by the coexistence of two magnetic field systems with opposite signs of magnetic helicity (Yokoyama et al. 2003; Chandra et al. 2010; Romano et al. 2011). Indeed, the magnetic helicity is a quantity with either a positive or a negative sign, which represents the right-handed linkage or the left-handed linkage of magnetic fluxes, respectively. This means that if positive and negative helicities coexist in a single domain, magnetic reconnection can cancel magnetic helicity by merging flux systems of opposite helicities. If it does, the reconnected field may relax towards a helicity-free state, and thus the free energy corresponding to magnetic helicity of mixed 
Table 1. List of the analysed ARs.

\begin{tabular}{lcccc}
\hline \hline AR NOAA & Start location & $\begin{array}{c}\text { Start date, } \\
\text { time (UT) }\end{array}$ & End location & $\begin{array}{c}\text { End date, } \\
\text { time (UT) }\end{array}$ \\
\hline 8210 & S17E42 & 1998 Apr. 28, 0000 & S17W37 & 1998 May 4, 0120 \\
8375 & N18E20 & 1998 Nov. 3, 0000 & N18W42 & 1998 Nov. 7, 1235 \\
9114 & N17E41 & 2000 Aug. 5, 2100 & N17W11 & 2000 Aug. 10, 2035 \\
9182 & N00E38 & 2000 Oct. 6, 0000 & N02E46 & 2000 Oct. 11, 2335 \\
9201 & N17E35 & 2000 Oct. 20, 1800 & N19W34 & 2000 Oct. 25, 2320 \\
9218 & N10E45 & 2000 Oct. 31, 0000 & N10W40 & 2000 Nov. 6, 1020 \\
\hline
\end{tabular}

signs should be released (Kusano et al. 1994). Therefore, we believe that the investigation of fluxes of different sign of magnetic helicity may be more useful than the total helicty flux in the estimation of an AR complexity and in its instability.

For this reason we considered the same dataset in this work as the one studied by Nindos et al. (2003) and by Pariat et al. (2006) to measure the horizontal velocity fields by the differential affine velocity estimator (DAVE) method (Schuck 2005) and to estimate the magnetic helicity flux distribution by the Pariat et al. (2005) method. Then we determined how fragmented the maps of helicity flux are to single out the number of magnetic features that are characterized by different signs of helicity flux and investigated its correlation with the flare and CME occurrence.

\section{Observations and analysis}

In order to study the ARs reported in Table 1, we used fulldisk line-of-sight magnetograms acquired by MDI/SOHO at $6767.8 \AA$ with a spatial resolution of 3.96 arcsec and a temporal resolution of $96 \mathrm{~min}$. We corrected all magnetograms for the angle between the magnetic field direction and the observer's line-of-sight. We aligned all subfields by applying a standard differential rotation rate (Howard et al. 1990) with a sampling of 1 arcsec (see Romano et al. 2011, for more details).

For each AR we followed this procedure:

- we determined the mean magnetogram corresponding to the average between two consecutive magnetograms (Fig. 1a);

- we measured the horizontal velocity fields with the DAVE method using a full-width-at-half maximum of the apodization window of 19.80 arcsec and a time interval of $96 \mathrm{~min}$ (Fig. 1b);

- we used the velocity fields to compute the maps of the magnetic helicity flux and the accumulation of magnetic helicity in the corona with the Pariat et al. (2005) method (Fig. 1c);

- we applied the labelling algorithm YAFTA (a single-pass "flux-ranked uphill gradient" algorithm described in Welsch \& Longcope 2003) on the helicity maps to group contiguous pixels into features (patches) that we defined to be convex groupings of 50 or more unipolar, contiguous pixels with a helicity flux density greater than $5 \times 10^{17} \mathrm{Mx}^{2} \mathrm{~cm}^{-2} \mathrm{~s}^{-1}$ in absolute value. An example of the features determined by this algorithm on a map of the helicity flux density of AR NOAA 8201 is shown in Fig. 1d;

- for each map we computed the total number of the identified patches, $N_{\mathrm{f}}$, and the absolute value of the difference between the number of patches of the two signs, $\left|N_{+}-N_{-}\right|$.

Nindos et al. (2003) selected the ARs reported in Table 1 because they were associated with halo CMEs that produced major geomagnetic storms between 1996 and 2000. Using the same data set, we investigated the temporal correlation between the flare and CME productivity of these ARs and the distribution of the helicity fluxes across them. On the basis of the reports of the Space Environment Center (http://www. swpc noaa. gov/) we considered the flares of $\mathrm{C}, \mathrm{M}$ and X GOES classes that occurred in the time intervals reported in Table 1 . We associated these events to a CME when they occurred less than one hour before or after the start time of the CMEs reported in the LASCO catalogue (http://cdaw.gsfc.nasa.gov/CME_ list/). In Table 1 we also give the corresponding locations on the solar disk of each AR at the beginning and at the end of the observations.

\section{Results}

In the first column of Fig. 2 we report the magnetic helicity accumulation for each AR during the selected time intervals. The time when the flares of GOES C, M and X classes and the associated CMEs occurred are indicated by vertical lines. These plots agree well with the results of Nindos et al. (2003), even if there are some slight differences because of the different methods used to measure the horizontal velocity fields and the magnetic helicity flux. We can distinguish three ARs that characterized by a prevalent positive flux of magnetic helicity (NOAA 8210, NOAA 8375 and NOAA 9182), two ARs with a negative flux (NOAA 9114 and NOAA 9218), and AR with an alternate trend of the sign of the flux. Therefore, if we consider the location of these ARs on the solar disk (see Table 1), only three out of six show a general trend of the helicity flux that agrees with the hemispheric magnetic helicity rule (see Pevtsov et al. 1995). We also note that NOAA 8210 and NOAA 9114 show a continuous increase of positive and negative magnetic helicity, respectively. On the other hand, NOAA 9182 and NOAA 9218 show a significant variation in the slope of the helicity accumulation only after half the observing time. We can see that the flare occurrence seems to be independent of the variations of the helicity transport rate. Although in AR NOAA 9201 the flares correspond to the peaks of the helicity accumulation and in AR NOAA 9182 the events are restricted only to the phase of helicity increase, in the other cases the flares and the CMEs occur during phases characterized by different slopes of the helicity accumulation curves.

The panels in the second column of Fig. 2 show the number of patches $N_{\mathrm{f}}$ for each AR identified by the YAFTA algorithm as a function of time. $N_{\mathrm{f}}$ can be considered an estimate of the fragmentation of the magnetic systems characterized by different magnetic helicity fluxes. Moreover, to show the distribution of $N_{\mathrm{f}}$ between the two signs of magnetic helicity, we report in the third column of Fig. 2 the panels regarding $\left|N_{+}-N_{-}\right|$vs. time. These plots highlight that the event occurrence seems to be temporally correlated with the variations of $N_{\mathrm{f}}$ and $\left|N_{+}-N_{-}\right|$.

For example, $N_{\mathrm{f}}$ for the AR NOAA 8210 varies between 20 and 60 . Most of the flares occur $60 \mathrm{~h}$ after the beginning of the observations when $N_{\mathrm{f}}$ is higher than 40 , while a small number of 


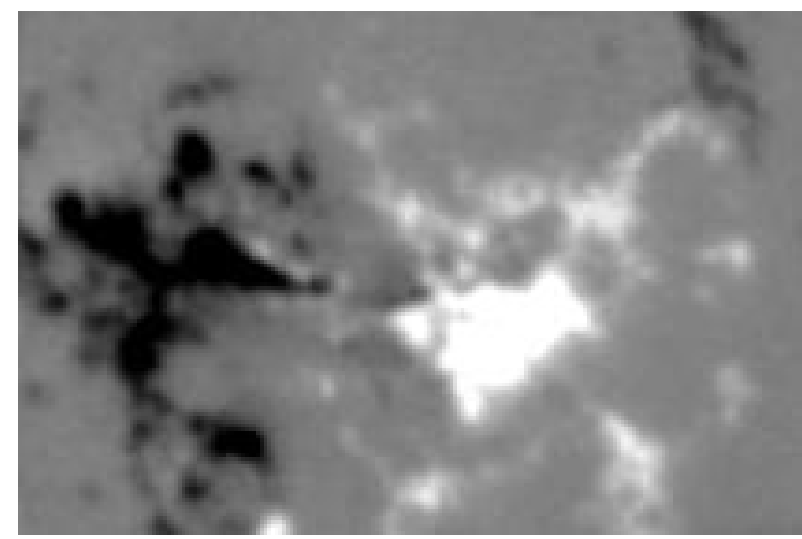

(a)

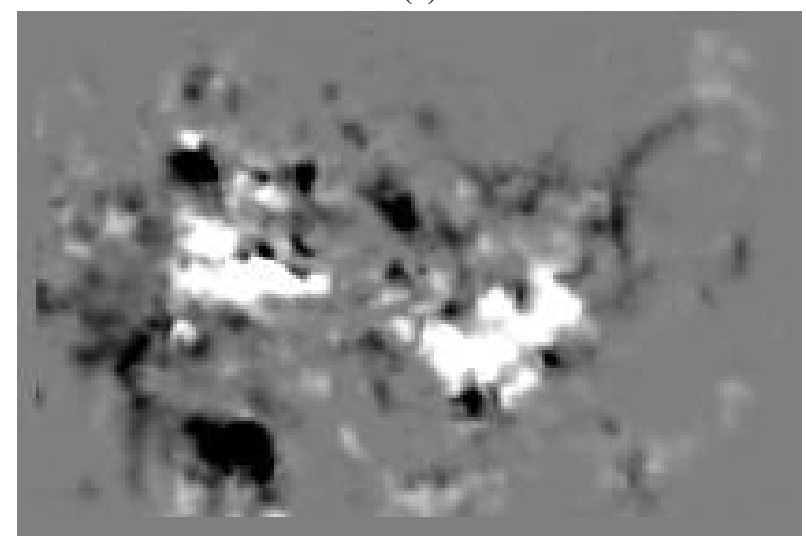

(c)

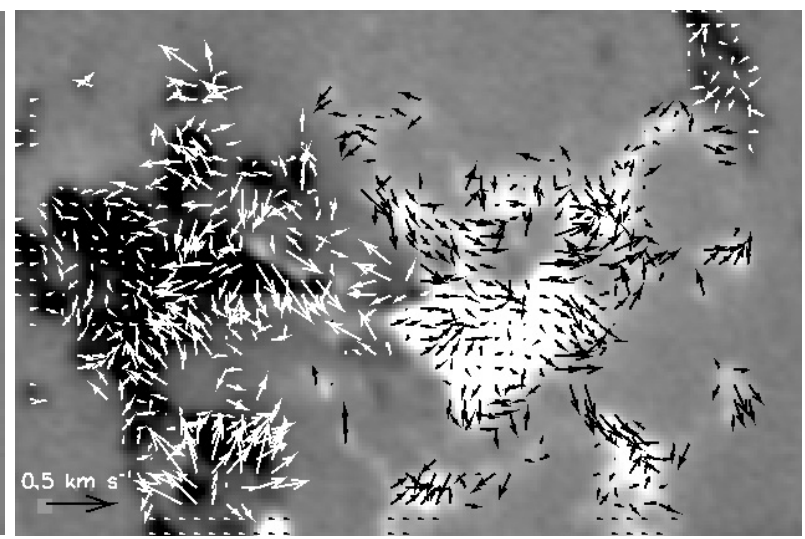

(b)

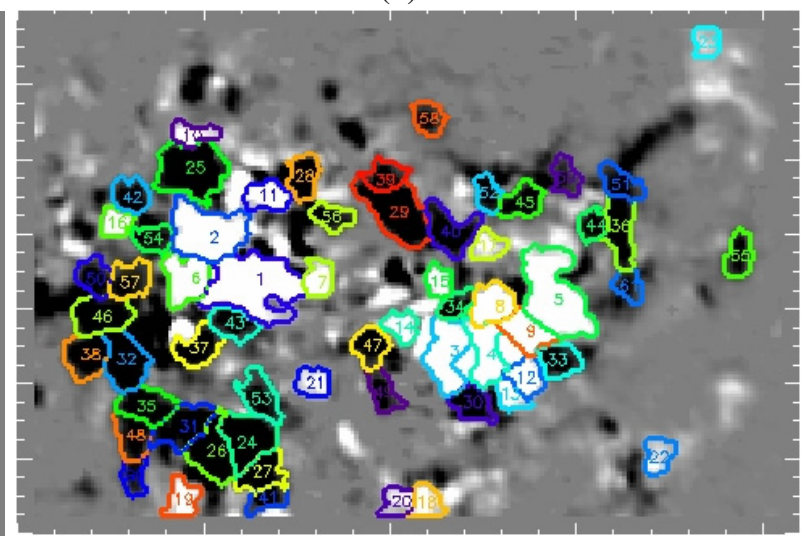

(d)

Fig. 1. Sample of the steps followed in the analysis of the MDI magnetograms taken on Oct. 20, 2000, at 00:00 UT and at 01:36 UT for AR 9201. a) Average magnetogram corresponding to 00:48 UT on Oct. 20,2000. Black and white correspond to negative and positive magnetic field, respectively. The saturation levels are $\pm 1000 \mathrm{G} ; \mathbf{b})$ the arrows indicate the horizontal velocities measured by means of the DAVE method. The background image is the average magnetogram showed in a); c) helicity flux density map computed with the method of Pariat et al. (2005). Black and white correspond to negative and positive helicity flux density, respectively. The saturation levels are $\left.\pm 5 \times 10^{18} \mathrm{Mx}^{2} \mathrm{~cm}^{-2} \mathrm{~s}^{-1} ; \mathbf{d}\right)$ elements of helicity flux determined by the YAFTA feature-tracking algorithm. The identified patches are numbered progresssively. The background image is the helicity flux density map showed in c) with saturation levels of $\pm 2 \times 10^{18} \mathrm{Mx}^{2} \mathrm{~cm}^{-2} \mathrm{~s}^{-1}$. The field of view is of about $210 \times 140$ arcsec .

flare occurs in the first part of the observations when $N_{\mathrm{f}}$ is less then 40 and no flare occurs from 45 to $70 \mathrm{~h}$ after the beginning of the observations when $N_{\mathrm{f}}$ reaches its minimum. On the other hand, most of the events occur when $\left|N_{+}-N_{-}\right|$is lower than 17 and the three most energetic events occur when $\left|N_{+}-N_{-}\right|$is lower than 10 , i.e. when the patches are many but are also widely distrubuted between the two opposite signs.

For AR NOAA 8375 the fragmentation increases continuously with helicity accumulation and event occurrence. After the first $20 \mathrm{~h}$ of observation, when the number of patches is clearly higher than 40, about 30 flares occur during the whole observing time. This very prolific AR is characterized by a low value of $\left|N_{+}-N_{-}\right|$. Only in the last $24 \mathrm{~h}$ of observation $\left|N_{+}-N_{-}\right|$increases and the number of events decreases.

The AR NOAA 9114 is characterized by a low activity: only three flares are registered during the observing time. In this case we do not see any peculiar trend in the fragmentation, but the first two events correspond to $\left|N_{+}-N_{-}\right|$equal to 5 and 3 , respectively.

An even better correlation is found for the NOAA 9182. For this AR both the helicity accumulation and the map fragmentation show an increase in the second half of the observing time, when six C GOES class flares occur. In this phase the number of patches increases to 80 but $\left|N_{+}-N_{-}\right|$remains $\leq 15$ at flare occurrence. No flare is observed when $N_{\mathrm{f}}$ is less than 20 .
The behaviour of AR NOAA 9201 is very interesting because the three flares observed in this AR occur about 31,42 and $69 \mathrm{~h}$ after the beginning of the observation when the the magnetic helicity flux changes sign, i.e. when the helicity accumulation changes its slope. These events occur in the first half of the observations, corresponding to the higher values of $N_{\mathrm{f}}$ and to low values of $\left|N_{+}-N_{-}\right|$. We can also note that in the period between the first two events the patches of one sign seem to prevail and no event is registered, although $N_{\mathrm{f}}$ is still high. No event is observed in the second half of the observations when on one hand the helicity accumulation increases till $1.5 \times 10^{42} \mathrm{Mx}^{2}$, but on the other hand the maps of the helicity flux appear less complex and more homogeneous, as the values of $N_{\mathrm{f}}$ indicate.

The AR NOAA 9218 is characterized by 4 C GOES class flares. Although the helicity accumulation does not show a steep variation during the first $60 \mathrm{~h}$ of observation, the number of features increases from 10 to 70 and three flares occur when $N_{\mathrm{f}}$ is higher than 40. Moreover, here the value of $\left|N_{+}-N_{-}\right|$has an opposite trend to the one of $N_{\mathrm{f}}$ when the flares occur.

If we consider only the flares associated with the CMEs (red vertical lines in Fig. 2), the relationship between the event occurrence and the higher values of $N_{\mathrm{f}}$ becomes more coherent in some cases. For example, if we consider the AR NOAA 9182, only one out of six flares could not be associated to a CME and it occurs just at the beginning of the increase phase of $N_{\mathrm{f}}$. 

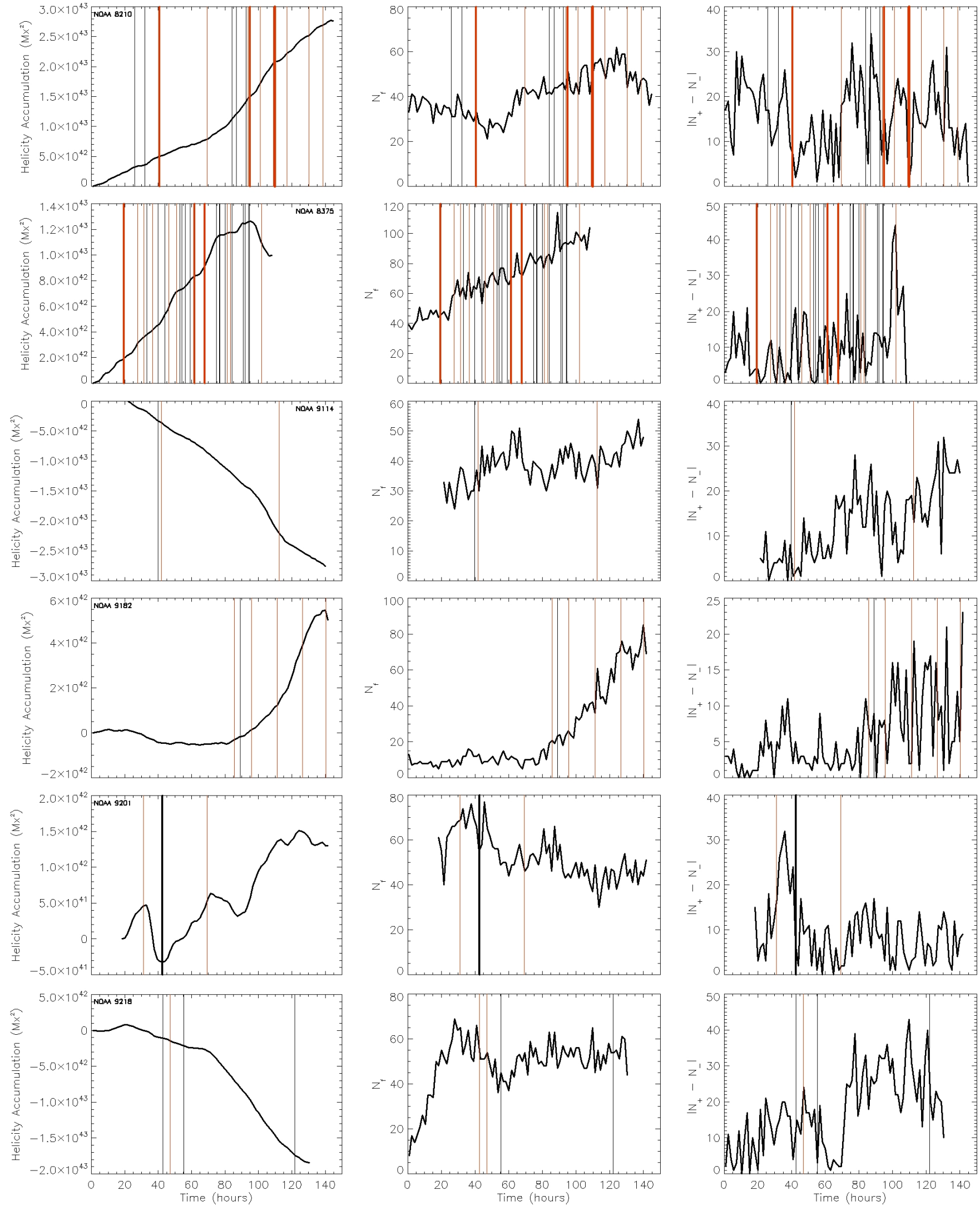

Fig. 2. First column: accumulation of the magnetic helicity vs. time. Second column: number of features determined by the YAFTA algorithm vs. time. Third column: absolute value of the difference between the numbers of positive and negative features vs. time. The plots of each row correspond to the AR reported in the plot of the first column. $t=0$ corresponds to 00:00 UT of the initial day reported in Table 1 (Col. 3). The vertical lines indicate the flare occurrence. The thickness of the vertical lines is equal to 1, 2 and 3 for flares of GOES C, M and X classes, respectively. The flares associated with CMEs are indicated in red. 
Finally, we note that all flares of $\mathrm{M}$ and $\mathrm{X}$ classes occur for $\left|N_{+}-N_{-}\right| \leq 10$ in all ARs.

\section{Discussion and conclusions}

The aim of the present paper is to show another aspect of the role played by the magnetic helicity transport from the convection zone to the corona: not only the intensity and the rate of the magnetic helicity accumulated in corona are important, but also its spatial distribution.

Although the ARs considered in the present study are characterized by different morphology, evolution, horizontal velocity behaviour, magnetic helicity flux, and accumulation, they show a good temporal correlation between the flare and CME occurrence and the fragmentation of the magnetic systems in terms of different magnetic helicity flux domains.

We derived the helicity flux density maps using the Pariat et al. (2005) method, which, although it is able to reduce spurious signals compared with the classical method (Chae et al. 2001), still presents some artefact polarities. As pointed out in Pariat et al. (2006), these fake polarities are caused by the computational limits of the measurement method and they are present when two different magnetic polarities are moving one with respect to the other and when a new flux tube is emerging, i.e. during every phase of an AR evolution, independently of its flare or CME activity. This means that the effect of the fake polarities can be considered a sort of noise in the estimation of the fragmentation of the patches in the helicity flux maps. Therefore, $N_{\mathrm{f}}$ might provide an overestimate of the total number of patches, but its variation is still a useful indicator of the degree of fragmentation. Moreover, because the fake polarities usually appear in pairs of opposite sign, the absolute value of the difference between the number of patches of the two signs, $\left|N_{+}-N_{-}\right|$, is independent of these spurious signals.

Therefore, even taking into account the aforementioned limits, our results indicate that the higher the number of features identified in the maps of the magnetic helicity flux, the higher the number of flares and CMEs, and that the lower the absolute value of the difference between the number of patches of the two signs, the higher the number of flares and CMEs.

We interpret this correlation as an indication of the important role played by the coexistence of systems that are characterized by opposite signs of magnetic helicity in triggering eruptive events, as already pointed out in previous observations of single events (Chandra et al. 2010; Romano et al. 2011) and as shown in the numerical simulations of Linton et al. (2001), Mok et al. (2001) and Kusano et al. (2004). Indeed, the interaction of magnetic fluxes with opposite helicity allows the release of the free energy because the field can relax to a state closer to a potential field through reconnection processes. For this reason, we believe that in the next future more efforts to investigate the spatial distribution should be made, instead of the simple trend of the magnetic helicity accumulation. This will help to better understand the relationship between the magnetic helicity and the release of the free magnetic energy in the corona. In this context, taking into account the magnetic connectivity in the computation of the magnetic helicity flux will be an important step to reach this goal (see Pariat et al. 2007).

Finally, we note that the fragmentation of the maps of magnetic helicity flux can be an indication of how monolitic the magnetic field of an AR is, i.e. how the twist and the writhe components of the magnetic helicity flux weight. Therefore, we think that additional efforts should be made also to distinguish the contribution of these components to know if one of them may be more efficient than the other to determine the conditions for the occurrence of the magnetic reconnection processes.

Acknowledgements. The authors wish to thank the referee for her/his comments and suggestions, which led to a sounder version of the manuscript. Thanks are also due to B. Welsh for the availability of the IDL programs of YAFTA. This work was supported by the European Commission through the SOLAIRE Network (MTRN-CT-2006-035484), by the Istituto Nazionale di Astrofisica (PRIN INAF 2010), by the Agenzia Spaziale Italiana (contract I/015/07/0), and by the Università degli Studi di Catania.

\section{References}

Chae, J. 2001, ApJ, 560, L95

Chandra, R., Pariat, E., Schmieder, B., Mandrini, C. H., \& Uddin, W. 2010, Sol. Phys., 261, 127

Howard, R. F., Harvey, J. W., \& Forgach, S. 1990, Sol. Phys., 130, 295

Kusano, K., Maeshiro, T., Yokoyama, T., \& Sakurai, T. 2004, ApJ, 610, 537

Linton, M. G., Dahlburg, R. B., \& Antiochos, S. K. 2001, ApJ, 553, 905

Mok, Y., Mikic, Z., \& Linker, J. 2001, ApJ, 555, 440

Nindos, A., Zhang, J., \& Zhang, H. 2003, ApJ, 594, 1033

November, L. J., \& Simon, G. W. 1988, ApJ, 333, 427

Pariat, E., Démoulin, P., \& Berger, M. A. 2005, A\&A, 439, 1191

Pariat, E., Nindos, A., Démoulin, P., \& Berger, M. A. 2006, A\&A, 452, 623

Pariat, E., Démoulin, P., \& Nindos, A. 2007, Adv. Space Res., 39, 1706

Pevtsov, A. A., Canfield, R. C., \& Metcalf, T. R. 1995, ApJ, 440, L109

Romano, P., Pariat, E., Sicari, M., \& Zuccarello, F. 2011, A\&A, 525, A13

Schuck, P. W. 2005, ApJ, 632, L53

Yokoyama, T., Kusano, K., Maeshiro, T., \& Sakurai, T. 2003, Adv. Space Res., 32, 1949

Welsch, B. T., \& Longcope, D. W. 2003, ApJ, 588, 620 